\title{
Análise de voz e comunicação oral de professores de curso pré-vestibular
}

\author{
Voice and oral communication analysis of preparatory school teachers
}

\author{
Andressa Consentino Vieira ${ }^{1}$, Mara Behlau ${ }^{2}$
}

\begin{abstract}
RESUMO
Objetivo: Realizar uma auto-avaliação com professores de curso pré-vestibular enfocando aspectos de voz, bem como analisar as características de voz, fala e recursos corporais utilizados em sala de aula. Métodos: Participaram deste estudo 40 professores do sexo masculino de uma empresa de cursos pré-vestibulares. Os docentes foram avaliados no local de trabalho por meio de dois questionários. O primeiro referente à auto-avaliação vocal e do ambiente de trabalho e o segundo referente à avaliação perceptivo-auditiva e visual de parâmetros corporais. A avaliação perceptivo-auditiva e visual foi realizada pela pesquisadora durante aulas-padrão de 60 minutos. Os parâmetros analisados foram divididos em aspectos de voz e fala. Os parâmetros referentes à análise dos recursos corporais foram pontuados em uma escala analógica graduada de 0 a 10, sendo que nesta escala o extremo esquerdo $(0)$ refere-se à inadequação e o extremo direito (10) à completa adequação. Resultados: A maioria dos professores relatou usar microfone (77,5\%) e apresentaram queixas de sinais e sintomas vocais, sendo os mais citados: falhas na voz, rouquidão, esforço para falar, secura na garganta e pigarro. Na análise perceptivo-auditiva observou-se que os professores apresentaram qualidade vocal discretamente alterada, sendo 35\% do tipo rouca. Os aspectos de fala e recursos corporais foram utilizados na maior parte do tempo de forma adequada. Conclusão: Os professores de curso pré-vestibular apresentaram elevada incidência de sintomas vocais, mesmo com a utilização de microfone. Utilizaram diversos recursos corporais na tentativa de potencializar o aprendizado, sendo que o uso de gestos, contato visual direcionado e interação verbal com os alunos foram as estratégias mais utilizadas em sala de aula.
\end{abstract}

Descritores: Docentes; Qualidade da voz; Sinais; Sintomas; Fala/fisiologia; Questionários/utilização; Doenças profissionais/ diagnóstico

\section{INTRODUÇÃO}

Professores são considerados profissionais da voz, pois dependem dela para o exercício de suas atividades profissionais. Podem estar inseridos no grupo que representa maior risco para o desenvolvimento de distúrbios vocais devido a particularidades do ambiente de trabalho e a demanda vocal elevada.

Condições ambientais e acústicas inadequadas, classes com número de alunos elevado, remuneração baixa e alta jornada de trabalho, são algumas das características laborais dos professores. Circunstâncias de caráter individual também devem ser consideradas como, predisposição orgânica, uso de substâncias irritativas ao trato respiratório e postura, entre outros. Estes fatores, somados ao despreparo vocal, podem limitar o desempenho ao longo da carreira letiva, deixando

Monografia apresentada como pré-requisito para a conclusão do Curso de Especialização em Voz no Centro de Estudos da Voz - CEV - São Paulo (SP), Brasil.

(1) Fonoaudióloga clínica, Especialista em Voz pelo Centro de Estudos da Voz - CEV - São Paulo, Brasil.

(2) Doutora, Professora do Centro de Estudos da Voz - CEV - São Paulo (SP), Brasil.

Endereço para correspondência: Andressa Consentino Vieira. R. Paula Ney, 480/91, Vila Mariana, São Paulo (SP), Brasil, CEP: 04107-021. E-mail: andy.fono@uol.com.br

Recebido em: 31/3/2008; Aceito em: 2/12/2008 dúvidas sobre os fatores prejudiciais a estabilidade da voz e com influência na qualidade de vida destes profissionais ${ }^{(1-3)}$.

Este grupo possui demanda vocal elevada por falar em forte intensidade, sobre ruído de fundo e por longos períodos de trabalho. O ruído dentro e fora da sala de aula exerce impacto considerável sobre a performance vocal, uma vez que muitos professores utilizam maior intensidade de voz que o habitual e referem incômodo devido à competição sonora. Desta forma, apresentam maior incidência de queixas vocais específicas e de desconforto físico em comparação a outras ocupações $^{(2-5)}$.

Recentes estudos epidemiológicos confirmam que os transtornos vocais são as lesões ocupacionais mais comuns entre professores dos EUA, o que afeta de diversas maneiras o desempenho profissional e a assiduidade no emprego ${ }^{(1,4)}$.

Professores de curso pré-vestibular, por razões profissionais, necessitam ser bons comunicadores, uma vez que devem transmitir grande quantidade de conteúdo e manter classes com número elevado de alunos sob controle e atentas. A comunicação em sala de aula envolve muitos aspectos, como a fala, o corpo e a voz para compor o discurso. Por esta razão, necessitam de competências comunicativas específicas. Para isso, utilizam diversos recursos vocais e corporais durante a aula, com o objetivo de despertar o interesse do aluno, facilitar a memorização e potencializar seu aprendizado. Portanto, o 
sucesso desta classe profissional pode depender em grande parte do seu perfil comunicativo.

Dentre os fatores envolvidos no processo de aprendizagem, está o domínio do conteúdo a ser transmitido, uso de recursos didáticos e interesse do aluno. É importante no ensino saber o que e como dizer aos alunos, saber se o objetivo da aula é oferecer formação e informação ou, ainda, motivar os alunos para determinada tarefa ${ }^{(6)}$.

Neste sentido, a voz é o principal meio de comunicação do professor, já que transmite conteúdos e características da personalidade. A voz deve ser saudável, ocorrer de maneira eficiente e sem esforço, ser interessante e clara, a fim de garantir a atenção do aluno. Assim como a voz, os movimentos do corpo transmitem informações sobre o indivíduo e complementam a mensagem. Na sala de aula a postura corporal e, principalmente da cabeça, pode refletir na aceitação visual e no funcionamento da musculatura da laringe ${ }^{(7)}$. Da mesma forma, deve-se levar em consideração a utilização de gestos que complementam a mensagem, a expressão facial durante o discurso e o contato visual direcionado ao aluno.

$\mathrm{O}$ uso de gestos e o contato visual direcionado refletem a personalidade e o comportamento do falante. Quando realizados de forma fluida e pertinente valorizam a apresentação, contribuindo para a transmissão da mensagem. O padrão articulatório com sons bem-definidos transmite ao ouvinte desejo de ser compreendido e clareza de idéias. Já uma articulação mal-definida pode indicar dificuldade na organização mental ou mesmo falta de vontade de se comunicar. Outro aspecto importante para a efetividade da mensagem é a velocidade de fala. Uma velocidade lenta desliga o ouvinte e passa impressão de lentidão de pensamento, ao passo que uma velocidade elevada pode refletir ansiedade e tensão além de não dar espaço para o interlocutor. Detalhes da expressão facial, dos gestos, da postura corporal e da voz criam um importante meio de comunicação que permeia a linguagem falada, favorecendo ou não o diálogo. Pessoas que sorriem com espontaneidade, que apresentam qualidade de voz agradável e demonstram receptividade com seus gestos, costumam atrair interlocutores ${ }^{(8)}$.

Considerando a exigência natural do perfil de comunicação destes profissionais, pode-se afirmar que qualquer alteração vocal ou desequilíbrio no uso dos recursos de comunicação, poderá comprometer sua atuação profissional. Desta forma, para a efetividade da assessoria fonoaudiológica e do tratamento vocal, faz-se necessário compreender as particularidades do docente, analisando como este utiliza a voz na sala de aula, pois estes dados viabilizam uma reflexão real do procedimento $\operatorname{vocal}^{(7)}$

Assim, o objetivo dessa pesquisa é caracterizar as condições de trabalho e analisar o perfil de comunicação de professores de curso pré-vestibular, identificando os recursos de voz, fala e corpo utilizados em sala de aula.

\section{MÉTODOS}

A pesquisa foi aprovada pelo Comitê de Ética do Centro de Estudos da Voz (n $\left.{ }^{\circ} 413-05\right)$ e todos os participantes assinaram o Termo de Consentimento Livre e Esclarecido. Participaram deste estudo 40 professores, do sexo masculino, de uma mesma empresa de cursos pré-vestibulares, com faixa etária de 19 a 65 anos, com média de 35 anos de idade. Considerou-se para critério de inclusão, todos os professores que fazem parte do corpo docente, que lecionassem de três a 40 aulas por semana com média de 21 aulas, correspondendo a menor e a maior grade de horários dentro da intuição pesquisada.

A pesquisa constou da aplicação de dois protocolos, sendo o primeiro de auto-avaliação vocal e investigação do ambiente de trabalho; e o segundo de análise perceptivo-auditiva da voz, fala, incluindo análise visual dos recursos corporais utilizados. O protocolo de auto-avaliação foi respondido individualmente pelos sujeitos antes das aulas. O questionário constou de 15 questões sobre: idade, carga horária semanal, disciplina ministrada, uso e tipo de microfone, presença de ar condicionado e poeira na sala de aula, hidratação, queixas e sintomas vocais atuais, realização de aquecimento e desaquecimento vocal, consulta a um fonoaudiólogo ou otorrinolaringologista e absenteísmo no trabalho por questões vocais. Em relação aos hábitos que influenciam na qualidade vocal, os professores foram questionados sobre: tabagismo e prática de outras atividades profissionais que necessitem do uso intensivo da voz.

As questões foram respondidas por meio de uma escala gradual de quatro pontos: nunca, às vezes, muitas vezes e sempre, que posteriormente foi reduzida para duas variáveis: sim e não. Incluiu-se na variável sim os aspectos às vezes, muitas vezes e sempre, e na variável não o aspecto nunca.

Cada professor foi avaliado pela mesma fonoaudióloga, durante aulas padrão de 50 minutos com média de 100 alunos por sala. O conteúdo semântico das aulas (mensagem verbal) não foi considerado. Os parâmetros de análise perceptivo-auditiva foram divididos em aspectos de voz e fala. Os aspectos de voz avaliados foram: qualidade vocal, ataque vocal, pitch, loudness, ressonância e coordenação pneumôfonica. Os aspectos de fala analisados foram: articulação, fluência, prosódia e uso de pausas no discurso.

$\mathrm{Na}$ análise visual os parâmetros selecionados foram: movimentação corporal, posição de cabeça, expressão facial, uso de gestos, contato visual e interação com os alunos. A avaliação foi realizada por meio de uma escala analógica visual de 10 centímetros. Nesta escala, o extremo esquerdo $(0$ a 3,9) significa ausência do parâmetro e de adequação, intermédio da escala (4 a 6,9), significa neutralidade do uso recurso, não comprometendo ou beneficiando a comunicação, e o extremo direito (7 a 10), presença completa de adequação. Todos os dados foram coletados pela pesquisadora e foi realizada análise estatística descritiva a partir do cálculo de médias simples e porcentagens.

\section{RESULTADOS}

Os resultados desta pesquisa estão apresentados nas Tabelas 1 a 4 .

Na Tabela 1 encontra-se a distribuição numérica e percentual dos dados referentes a critérios de auto-avaliação dos professores.

A Tabela 2 apresenta a distribuição numérica e percentual da frequência e tipos de sinais e sintomas referidos pelos professores. 
Tabela 1. Resultados da auto-avaliação vocal e do ambiente de trabalho dos professores

\begin{tabular}{|c|c|c|c|c|}
\hline & \multicolumn{2}{|c|}{ Sim } & \multicolumn{2}{|c|}{ Não } \\
\hline & $\mathrm{N}$ & $\%$ & $\mathrm{~N}$ & $\%$ \\
\hline \multicolumn{5}{|l|}{ Dados vocais } \\
\hline Queixas vocais atuais & 11 & 27,5 & 29 & 72,5 \\
\hline Queixas vocais anteriores & 22 & 55 & 18 & 45 \\
\hline Absenteísmo por problemas de voz & 11 & 27,5 & 29 & 72,5 \\
\hline Procuraram Fono/ORL & 7 & 17,5 & 33 & 82,5 \\
\hline Realizam aquecimento vocal & 10 & 25 & 30 & 75 \\
\hline Realizam desaquecimento vocal & 2 & 5 & 38 & 95 \\
\hline Outra atividade profissional com voz & 17 & 42,5 & 23 & 57,5 \\
\hline Hidratação & 19 & 47,5 & 21 & 52,5 \\
\hline Fumam & 12 & 30 & 28 & 70 \\
\hline \multicolumn{5}{|l|}{ Caracterização do trabalho } \\
\hline Presença de ar condicionado & 40 & 100 & 0 & 0 \\
\hline Presença de poeira & 37 & 92,5 & 3 & 7,5 \\
\hline Uso de microfone & 31 & 77,5 & 9 & 22,5 \\
\hline
\end{tabular}

Tabela 2. Distribuição numérica e percentual da frequência e tipo de sinais e sintomas referidos pelos professores

\begin{tabular}{lcc}
\hline & N & $\%$ \\
\hline Quantidade de sinais e sintomas & 12 & 30 \\
- / 2 & 1 & 2,5 \\
3 & 27 & 67,5 \\
$4 /+$ & & \\
Tipos de sinais e sintomas & 9 & 22,5 \\
Cansaço vocal & 21 & 52,5 \\
Falhas & 14 & 35,0 \\
Dificuldade de projeção & 21 & 52,5 \\
Rouquidão & 21 & 52,5 \\
Esforço para falar & 15 & 37,5 \\
Dor na laringe & 26 & 65,0 \\
Secura na garganta & 21 & 52,5 \\
Pigarro & 10 & 25,0 \\
RGE & 15 & 37,5 \\
ICPF & 7 & 17,5 \\
Dificuldade de modulação &
\end{tabular}

Legenda: RGE = refluxo gastroesofágico; ICPF = incoordenação pneumofonoarticulatória
Na Tabela 3 observa-se a distribuição numérica, percentual e absoluta dos recursos corporais utilizados em sala de aula.

A Tabela 4 refere-se à distribuição numérica e percentual dos dados da análise perceptivo-auditiva dos aspectos de voz e fala durante as aulas.

\section{DISCUSSÃO}

Com relação ao ambiente de trabalho, 92,5\% (37) relata que possui contato com a substância de pó de giz e $100 \%$ (40) refere uso diário do ar condicionado (Tabela 1). O uso do refrigerador de ar foi justificado devido à amplitude das salas e a necessidade de manter janelas fechadas para facilitar a concentração dos alunos. Estas condições contribuem para o ressecamento da mucosa da laringe e favorecem o aparecimento de quadros alérgicos, prejudicando o desempenho vocal ao longo das aulas ${ }^{(2,4,9)}$.

Quanto ao hábito de hidratação (Tabela 1), 52,5\% (21) não ingere líquidos durante o período em que lecionam, muitos para não interromper a aula por necessidade de urinar. A falta de lubrificação também pode ser observada no relato de sintomas vocais e laríngeos, quando 65\% (26) dos professores referem sintoma de secura na laringe e $53 \%$ (21) de pigarro (Tabela 2). O pigarro também pode ser associado ao tabagismo,

Tabela 3. Média, distribuição percentual e absoluta dos recursos corporais utilizados em sala de aula

\begin{tabular}{lccccccc}
\hline Recursos corporais & Média & \multicolumn{2}{c}{ Entre 0 e 3,9 } & \multicolumn{2}{c}{ Entre 4 e 6,9 } & \multicolumn{2}{c}{ Entre 7 e 10 } \\
\cline { 3 - 7 } & & $\mathrm{N}$ & $\%$ & $\mathrm{~N}$ & $\%$ & $\mathrm{~N}$ & $\%$ \\
\hline Movimentação corporal & 7,3 & 4 & 10,0 & 7 & 17,5 & 29 & 72,5 \\
Posição de cabeça & 6,7 & 7 & 17,5 & 9 & 22,5 & 24 & 60,0 \\
Expressão facial & 7,7 & 2 & 5,0 & 9 & 22,5 & 29 & 72,5 \\
Uso de gestos & 8,3 & 1 & 2,5 & 4 & 10,0 & 35 & 87,5 \\
Contato visual & 8,6 & 0 & 0,0 & 4 & 10,0 & 36 & 90,0 \\
Interação com o público & 8,8 & 0 & 0,0 & 1 & 2,5 & 39 & 97,5 \\
Psicodinâmica da comunicação & 7,7 & 1 & 2,5 & 8 & 20,0 & 31 & 77,5 \\
\hline
\end{tabular}

Legenda: $\mathrm{N}$ = número; \% = porcentagem 
Tabela 4. Parâmetros da análise perceptivo-auditiva dos aspectos de voz e fala utilizados durante as aulas

\begin{tabular}{|c|c|c|}
\hline Aspectos de voz e fala & $\mathrm{N}$ & $\%$ \\
\hline \multicolumn{3}{|l|}{ Tipos de voz } \\
\hline Adaptada & 20 & 50 \\
\hline Alterada & 20 & 50 \\
\hline - Hiponasal & 4 & 10 \\
\hline - Feminilizada & 1 & 2,5 \\
\hline - Rouca & 14 & 35 \\
\hline - Tensa & 1 & 2,5 \\
\hline \multicolumn{3}{|l|}{ Ressonância } \\
\hline Equilibrada & 23 & 57,5 \\
\hline Alterada & 17 & 42,5 \\
\hline - Nasal & 2 & 5 \\
\hline - Oral & 7 & 17,5 \\
\hline - Laríngea & 8 & 20 \\
\hline \multicolumn{3}{|l|}{ Ataque vocal } \\
\hline Isocrônico & 28 & 70 \\
\hline Brusco & 12 & 30 \\
\hline \multicolumn{3}{|l|}{ Pitch } \\
\hline Adaptado & 22 & 55 \\
\hline Alterado & 18 & 45 \\
\hline - Agudo & 5 & 12,5 \\
\hline - Grave & 13 & 32,5 \\
\hline \multicolumn{3}{|l|}{ Loudness } \\
\hline Adaptada & 31 & 77,5 \\
\hline Alterada & 9 & 22,5 \\
\hline - Diminuída & 2 & 5 \\
\hline - Aumentada & 7 & 17,5 \\
\hline \multicolumn{3}{|c|}{ Coordenação pneumôfonica } \\
\hline Coordenada & 25 & 62,5 \\
\hline Incoordenada & 15 & 37,5 \\
\hline \multicolumn{3}{|l|}{ Velocidade de fala } \\
\hline Adaptada & 31 & 77,5 \\
\hline Alterado & 9 & 22,5 \\
\hline - Acelerada & 8 & 20 \\
\hline - Lenta & 1 & 2,5 \\
\hline \multicolumn{3}{|l|}{ Pausas } \\
\hline Adaptadas & 33 & 82,5 \\
\hline Alteradas & 7 & 17,5 \\
\hline - Longas & 2 & 5,0 \\
\hline - Curtas & 5 & 12,5 \\
\hline \multicolumn{3}{|l|}{ Padrão articulatório } \\
\hline Adaptado & 29 & 72,5 \\
\hline Alterado & 11 & 27,5 \\
\hline - Imprecisão & 3 & 7,5 \\
\hline - Subarticulação & 7 & 17,5 \\
\hline - Sobrearticulação & 1 & 2,5 \\
\hline \multicolumn{3}{|l|}{ Prosódia } \\
\hline Adaptada & 21 & 52,5 \\
\hline Alterado & 19 & 47,5 \\
\hline - Excessiva & 9 & 22,5 \\
\hline - Restrita & 7 & 17,5 \\
\hline - Repetitiva & 3 & 7,5 \\
\hline
\end{tabular}

uma vez que o cigarro aumenta a secreção viscosa na laringe, favorecendo o ato de pigarrear ${ }^{(8)}$. Neste estudo, $30 \%$ (12) da amostra é fumante (Tabela 1).

Com relação ao uso do microfone (Tabela 1), os resultados mostram que 77,5\% (31) faz uso do aparelho de amplificação, sendo os tipos de lapela $40 \%$ (16) e de mão 32,5\% (13), os mais utilizados. $\mathrm{O}$ uso do microfone tem se mostrado uma estratégia eficiente para o conforto e longevidade vocal do profissional da voz, além de ser um recurso alternativo para alterações vocais ${ }^{(4,10)}$. A utilização da amplificação sonora pode ter contribuído para o conforto fonatório mesmo em quadros de infecções de vias áreas superiores e alterações na qualidade vocal, uma vez que 72,5\% (29) dos professores não apresentam queixas em relação à voz e apenas $27,5 \%$ (11) necessitou afastar-se de suas atividades por problemas vocais. Entretanto, não é um recurso exclusivo de prevenção vocal, uma vez que 55\% (22) já apresentaram problemas vocais ao longo da carreira letiva.

Em relação à prática de exercícios vocais, $75 \%$ (30) não realiza aquecimento vocal e $95 \%$ (38) não realiza desaquecimento vocal (Tabela 1). Estes dados podem ser associados à falta de informação sobre os benefícios destes exercícios, bem como a dificuldade apresentada em relacionar um problema de voz a um problema de saúde ${ }^{(11-12)}$, fato que se evidencia em relação à busca por auxilio profissional. Observou-se que $82,5 \%$ (33) nunca procurou auxilio fonoaudiológico ou otorrinolaringológico devido a questões vocais (Tabela 1), sendo que $67,5 \%$ (27) dos professores relataram mais de quatro sintomas vocais, sendo os mais referidos, falhas na voz $(52,5 \%)$, rouquidão $(52,5 \%)$, esforço para falar $(52,5 \%)$, secura na garganta $(65 \%)$ e pigarro $(52,5 \%)$. Estes dados confirmam a falta de informação em relação à promoção da saúde da voz, que faz com que os professores só procurem auxilio especializado quando os sintomas ou alterações estão evidentes ${ }^{(13-14)}$. Com relação ao índice elevado de sintomas vocais (Tabela 2), este pode estar associado ao uso constante do ar condicionado, à presença de pó de giz e à falta de hidratação, sendo que a somatória destes fatores negativos pode potencializar o risco de aparecimento de disfonias. Esses dados também podem revelar a grande possibilidade do professor usar inadequadamente sua voz em suas atividades, fato que deve ser considerado como alerta para esta população ${ }^{(3-4)}$.

Em relação ao exercício de atividades profissionais paralelas à docência, 42,5\% (17) referiu realizá-las; sendo as mais referidas, medicina, canto, radialismo, palestras, coordenação e autoria de materiais didáticos. Portanto nota-se que o perfil destes professores é voltado para o aprimoramento da comunicação oral e escrita.

Os resultados referentes aos recursos corporais (Tabela 3 ) mostram que a população estudada possui elevada conscientização referente à apresentação em público, uma vez que não há treinamento de performance comunicativa na instituição pesquisada. Observou-se que 72,5\% (29) dos docentes movimentam-se com naturalidade; $60 \%$ (24) apresenta uma relação retilínea com o tronco por meio de um alinhamento da coluna vertebral; 72,5\% (29) apresenta expressão coerente ao conteúdo do discurso e ao contexto da comunicação; $90 \%$ realiza o contato visual de forma adequada, direcionando 
o olhar e contemplando a maioria dos alunos; $87,5 \%$ (35) utiliza gestos com adequação ${ }^{(4,8)}$. Com relação à interação com o público, observa-se que $97,5 \%$ (39) faz contato verbal com os alunos permitindo que os mesmos façam perguntas, além de inserirem estratégias durante as aulas como, músicas para auxilio de memorização, participações em experiências práticas e observações verbais feitas em vídeos e animações.

Os dados obtidos referentes à qualidade vocal, mostram que 50\% (20) apresentam voz alterada, sendo a maior parte do tipo rouca $35 \%$ (14). Tais alterações podem ser justificas pelo uso de ajustes individuais de laringe e faringe, tabagismo, mau uso da voz, além de quadros crônicos de infecções de vias áreas superiores, que podem ser desencadeados pelo uso de ar condicionado e do contato com pó de giz durante as aulas ${ }^{(6,8)}$. Com relação à ressonância (Tabela 4), 57,5\% (23) apresenta uso equilibrado e $42,5 \%$ (17) desequilibrado, sendo $5 \%$ (2) nasal; $17,5 \%$ (7) oral e 20\% (8) laríngea. Pode-se atribuir as alterações de ressonância aos ajustes fonatórios do docente e ao despreparo para o uso do microfone, uma vez que muitos apresentam uso excessivo da laringe, mesmo utilizando o aparelho de amplificação.

Houve predominância de loudness adaptada em 77,5\% (31) dos sujeitos, ataque vocal isocrônico em 70\% (28) e coordenação pneufonoarticulatória em 62,5\% (25) na maior parte do tempo. Em relação ao pitch (Tabela 4) observou-se que 55\% (22) da amostra apresenta pitch adaptado e $45 \%$ (18) alterado, sendo $12,5 \%$ (5) agudo e 32,5\% (13) grave. A presença do pitch grave pode ser associada à qualidade vocal rouca, presente em $35 \%$ (14) dos professores e ao tabagismo presente em $30 \%$ (12).

Houve predomínio de adequação referente aos aspectos de fala (Tabela 4), sendo que 77,5\% (31) apresenta velocidade de fala adaptada, 82,5\% (33) utiliza pausas coerentes ao discurso; e 72,5\% (29) possui padrão adaptado. Em relação à modulação, $52,5 \%$ (21) mostra-se adaptada e 47,5\% (19) alterada, sendo $22,5 \%$ (9) do tipo excessiva; $17,5 \%$ (7) restrita; e 7,5\% (3) repetitiva. Isso revela que a maioria dos professores de curso pré-vestibular apresenta controle da dinâmica fonatória, clareza de idéias e vontade de ser compreendido, passando credibilidade ao aluno ${ }^{(8)}$.

Nota-se que professores de curso pré-vestibular apresentam particularidades em seu ambiente de trabalho, como uso sistemático do ar condicionado e do aparelho de amplificação sonora, realidade muito diferente da maioria dos docentes de ensino médio e fundamental ${ }^{(15-16)}$. Entretanto, observou-se elevado índice de sintomas vocais nesta população, fato que pode gerar risco elevado para o desenvolvimento de alterações vocais, uma vez que o professor permanece em suas atividades mesmo com a presença do desconforto fonatório. Os parâmetros referentes à avaliação perceptivo auditiva da voz e da fala encontraram-se adaptados na maioria da amostra. Já os recursos de comunicação não-verbal utilizados durante as aulas, reforçaram que os professores de curso pré-vestibular apresentam bom domínio de recursos corporais. Deve-se destacar que estes dados referem-se a uma pequena amostra de professores de uma determinada instituição e, portanto sugere-se que outros trabalhos sejam efetuados para a ampliação da literatura e para a melhor compreensão das particularidades destes profissionais.

\section{CONCLUSÃo}

Os professores de curso pré-vestibular possuem carga horária de trabalho variada, lecionam em ambiente refrigerado e com amplificação sonora. Apresentam elevado índice de sintomas vocais, sendo os mais referidos: falhas na voz, rouquidão, esforço para falar, secura na garganta e pigarro. Apresentam adequação nos parâmetros de voz e fala e no uso de recursos não verbais utilizados em sala de aula, sendo o uso de gestos, o contato visual direcionado e a interação com os alunos, os mais utilizados.

\begin{abstract}
Purpose: To carry out a self-evaluation with preparatory school teachers regarding vocal aspects, as well as to analyze characteristics of voice, speech and corporal resources used during classes. Methods: Forty male teachers from a preparatory school were selected for this study. They were assessed in their workplace using two questionnaires. The first questionnaire regarded the workplace and vocal self-evaluation, and the second concerned visual and auditory-perceptual assessment of corporal parameters. The visual and auditory-perceptual assessment was carried out by the examiner during regular 60-minute classes. The parameters analyzed were divided into voice and speech aspects. The parameters that regarded corporal resources analysis were scored on an analogical scale graduated from 0 to 10 , where the far left (0) referred to inadequacy, and the far right (10), to complete adequacy. Results: Most teachers $(77.5 \%)$ used microphone during classes and reported vocal signs and symptoms, among which the most cited were: voice failures, hoarseness, speech effort, throat dryness and accumulation of mucus in the throat. Auditory-perceptual analysis showed that teachers had slightly altered vocal quality, being $35 \%$ characterized as hoarse. Speech and corporal resources were used adequately most of the time. Conclusion: Prep-school teachers showed high incidence of vocal symptoms even when using microphone. They used of several corporal resources in order to enhance learning, and use of gestures, directed eye contact, and verbal interaction with students were the most used strategies during classes.
\end{abstract}

Keywords: Faculty; Vocal quality; Signs; Symptoms; Speech/physiology; Questionnaires/utilization; Occupational diseases/diagnosis 


\section{REFERÊNCIAS}

1. Roy N. Teachers with voice disorders: recent clinical trials research. The ASHA Leader. 2005;10(5):8-9,11.

2. Gonçalves CGO, Penteado RZ, Silvério KCA. Fonoaudiologia e saúde do trabalhador: a questão da saúde vocal do professor. Saúde Rev. 2005;7(15):45-51.

3. Williams NR. Occupational groups at risk of voice disorders: a review of the literature. Occup Med (Lond). 2003;53(7):456-60.

4. Roy N, Gray SD, Simon M, Dove H, Corbin-Lewis K, Stemple JC. An evaluation of the effects of two treatment approaches for teachers with voice disorders: a prospective randomized clinical trial. J Speech Lang Hear Res. 2001;44(2):286-96.

5. Vilkman E. Voice problems at work: A challenge for occupational safety and health arrangement. Folia Phoniatr Logop. 2000;52(1-3):120-5.

6. Behlau M, Dragone MLS, Nagano L. A voz que ensina: o professor e a comunicação oral em sala de aula. Rio de Janeiro: Revinter; c2004.

7. Dragone MLOS. Novos caminhos para os estudos sobre a voz do professor. Rev Fonoaudiol Brasil. 2001;1(1):43-50.

8. Kyrillos LR, organizadora. Expressividade: da teoria à prática. Rio de Janeiro: Revinter; 2005.

9. Schwarz K, Cielo CA. A voz e as condições de trabalho de professores de cidades pequenas do Rio Grande do Sul. Rev Bras Fonoaudiol. 2005;10(2);83-90.
10. Roy N, Weinrich B, Gray SD, Tanner K, Toledo SW, Dove H, et al. Voice amplification versus vocal hygiene instruction for teachers with voice disorders: a treatment outcomes study. J Speech Lang Hear Res. 2002;45(4):625-38. Comment in: J Speech Lang Hear Res. 2004;47(2):353-7; author reply 358-65.

11. Zambon FC. Sintomas vocais, hábitos e condições de trabalho dos professores atendidos no SINPRO-SP [monografia]. São Paulo: Centro de Estudos da Voz - CEV; 2005.

12. Penteado ZR. Relações entre saúde e trabalho docente: percepções de professores sobre saúde vocal. Rev Soc Bras Fonoaudiol. 2007;12(1):1822.

13. Secretaria de Estado da Saúde de São Paulo. Centro de Referência em Saúde do Trabalhador. Coordenadoria de Controle de Doenças. Distúrbios de voz relacionados ao trabalho. Bol Epidemiol Paul. 2006;3(26):16-22.

14. Grillo MHMM, Penteado RZ. Impacto da voz na qualidade de vida de professore (a)s do ensino fundamental. Pró-Fono. 2005;17(3):321-30.

15. Dragone MLS, Behlau M. A fonoaudiologia brasileira e a voz do professor - olhares científicos no decorrer do tempo. Fonoaudiol Brasil. 2007; 4(2):6-9.

16. Mariano SA. Perfil vocal do professor de cursinho: análise perceptivoauditiva e acústica [monografia]. São Paulo: Centro de Estudos da Voz - CEV; 2004. p.27-35. 